\title{
Approximate Methods for the Calculation of Plasma Internal Inductance for Circular Cross Section Tokamak
}

\author{
M. Asif \\ Department of Physics, COMSATS Institute of Information Technology, Lahore, Pakistan \\ Email: dr.muha.asif@gmail.com
}

Received December 25, 2012; revised January 20, 2013; accepted February 18, 2013

Copyright (C) 2013 M. Asif. This is an open access article distributed under the Creative Commons Attribution License, which permits unrestricted use, distribution, and reproduction in any medium, provided the original work is properly cited.

\begin{abstract}
Calculation of Plasma internal inductance $\left(l_{i}\right)$ is essential in Tokamak plasma research. Much more plasma parameters such as the plasma current density profile, magnetohydrodynamics instability, and plasma energy confinement time can be determined by using this parameter. Discrete poloidal magnetic probes along with the diamagnetic loop can be utilized in measurement of the Plasma internal inductance $\left(l_{i}\right)$. In this paper Plasma internal inductance $\left(l_{i}\right)$ is studied by theoretical and experimental approach for HT-7 Tokamak plasmas. The results of two methods are in good agreement with each other.
\end{abstract}

Keywords: Tokamak; Plasma Internal Inductance; Magneto-Hydrodynamic

\section{Introduction}

The most advanced approach towards the achievement of the relevant fusion reactor parameters is the confinement of a plasma within a magnetic field in a so-called tokamak [1,2]. Tokamaks are one of the leading candidates for magnetically confined fusion owing to their good particle and energy confinement.

Plasma inductance control is an essential profile control tool for Tokamaks that can be used to extend pulse duration [2], access to advanced regimes, reduce vertical instability growth rate, and improve experiment reproducibility.

In this work we present measurement of Plasma internal inductance $\left(l_{i}\right)$ [3-5], which is essential in Tokamak plasma research. Much more plasma parameters such as the plasma current density profile, magnetohydrodynamics instability, and plasma energy confinement time can be determined by using this parameter. Discrete poloidal magnetic probes along with the diamagnetic loop can be utilized in measurement of the Plasma internal inductance $\left(l_{i}\right)$. In this paper we present theoretical calculation of Plasma internal inductance $\left(l_{i}\right)$ for HT-7 Tokamak [1, 2], (see Table 1). The Plasma internal inductance $\left(l_{i}\right)$ is also studied for Tokamaks [3-5] by theoretical and experimental approach.

\section{Theoretical Calculation of Plasma Internal Inductance $\left(l_{i}\right)$}

The internal inductance of the plasma per unit length normalized to $\mu_{0} / 4 \pi$ is obtained from the conservation of zeroth order magnetic energy $[3,4]$ as:

$$
l_{i}=\frac{L_{i} / 2 \pi R_{0}}{\mu_{0} / 4 \pi}=\frac{2}{\mu_{0}^{2} I_{p}^{2} R_{0}} \int B_{\theta}^{2}(r) \mathrm{d}^{3} V
$$

where $I_{p}$ is the plasma current. $R$ and $r$ are the HT-7 Tokamak $[1,2]$ major and minor radii. For typical profile of the poloidal field which corresponds to flat plasma current density profile $J_{0}$ (usually is the case for low beta plasma Tokamak) is:

$$
\begin{aligned}
& J=J_{0} \rightarrow B_{\theta}=\frac{B_{\theta a} r}{a}, r<a \\
& J=0 \rightarrow B_{\theta}=\frac{B_{\theta a} r}{a}, a<r \leq b
\end{aligned}
$$

where $B_{\theta a}=\frac{\mu_{0} I_{p}}{2 \pi a}, a$ and $b$ are the plasma and chamber radiuses respectively.

Therefore, the first approximate value for the internal inductance can be easily obtained by substituting Equation (2) into Equation (1): 
Table 1. Parameter of the HT-7 Tokamak.

\begin{tabular}{cc}
\hline Parameter & Value \\
\hline Major Radius & $1.22 \mathrm{~m}$ \\
Minor Radius & $0.25 \mathrm{~m}$ \\
Toroidal Field & $1-2.5 \mathrm{~T}$ \\
Plasma Current & $100-250 \mathrm{kA}$ \\
Discharge Time & $\sim 300 \mathrm{~s}$ \\
Electron Density & $1-6 \times 10^{19} \mathrm{~m}^{-3}$ \\
\hline
\end{tabular}

$$
l_{i 1}=\frac{1}{2}-2 \ln \frac{a}{b}
$$

This relation for HT-7 Tokamak [1,2] plasmas parameters (see Table 1) is equal to 0.727 .

The second approximate value for the internal inductance can be easily obtained from the well-known Bennett current density profile $[3,4]$, as:

$$
\begin{aligned}
& J=\frac{I_{p} a^{2}}{\pi\left(r^{2}+a^{2}\right)^{2}}, r \leq a \\
& J=0, a<r<b \\
& B_{\theta}=\frac{\mu_{0} I_{p}}{2 \pi}\left[\frac{r}{r^{2}+a^{2}}\right], r \leq a \\
& B_{\theta}=\frac{\mu_{0} I_{p}}{4 \pi r}, a<r<b
\end{aligned}
$$

Then the second approximate value for the internal inductance can be easily obtained:

$$
l_{i 2}=\frac{1}{2}\left(\ln \frac{4 b}{a}-1\right)
$$

This relation for HT-7 Tokamak [1,2] plasmas parameters (see Table 1) is equal to 0.250 .

In general case, for the large aspect ratio and circular plasma, the current density distribution is [6], given as

$$
\begin{aligned}
& J=J(0)\left(1-\frac{r^{2}}{a^{2}}\right)^{v}, r<a \\
& J=0, a<r<b
\end{aligned}
$$

The Poloidal magnetic field profile can be obtained:

$$
\begin{aligned}
& B_{\theta}=\frac{\mu_{0} J(0) a^{2}}{2(v+1) r}\left(1-\left(1-\frac{r^{2}}{a^{2}}\right)^{v+1}\right), r \leq a \\
& B_{\theta}=\frac{\mu_{0} J(0) a^{2}}{2(v+1) r}, a<r<b
\end{aligned}
$$

where

$$
J(0)=\frac{I_{p} q(a)}{\pi a^{2} q(0)}=\frac{I_{p}(v+1)}{\pi a^{2}}
$$

The geometrical parameters of the HT-7 Tokamak are shown in Table 1, where $q(a)$ and $q(0)$ are the edge and central safety factor respectively. The values of the internal inductance can be easily obtained by substituting Equation (8) into Equation (1) as a function of the $v$.

The results are given in Table 2 and Figure 1.

\section{Plasma Internal Inductance $\left(l_{i}\right)$ by Discrete Poloidal Probes along with the Diamagnetic Loop}

But the final approximate method for the measurement of plasma internal inductance on HT-7 Tokamak is experimental method [5]. Discrete poloidal magnetic probes along with the diamagnetic loop can be utilized in measurement of the Plasma internal inductance $\left(l_{i}\right)$ In this method [5] firstly Shafranov parameter is obtained from the magnetic probe measurements and then the values of the poloidal beta which obtained by the diamagnetic loop is subtracted from it as:

$$
l_{i 4}=2\left(\Lambda-\beta_{p}+1\right)
$$

\section{Conclusions}

We have described calculation of Plasma internal inductance $\left(l_{i}\right)$ [3-5], which is essential in Tokamak plasma

Table 2. Dependence of Internal inductance $\left(l_{i}\right)$ to the values of $v$ for HT-7 Tokamak plasmas.

\begin{tabular}{cccccc}
\hline Sr. No. & $v$ & $\begin{array}{c}\text { Internal } \\
\text { inductance }\left(l_{i}\right)\end{array}$ & Sr. No. & $v$ & $\begin{array}{c}\text { Internal } \\
\text { inductance }\left(l_{i}\right)\end{array}$ \\
\hline 1 & 0 & 0.821 & 7 & 6 & 2.073 \\
2 & 1 & 1.347 & 8 & 7 & 2.183 \\
3 & 2 & 1.461 & 9 & 8 & 2.303 \\
4 & 3 & 1.596 & 10 & 9 & 2.496 \\
5 & 4 & 1.713 & 11 & 10 & 2.699 \\
6 & 5 & 1.863 & 12 & 11 & 2.823 \\
\hline
\end{tabular}

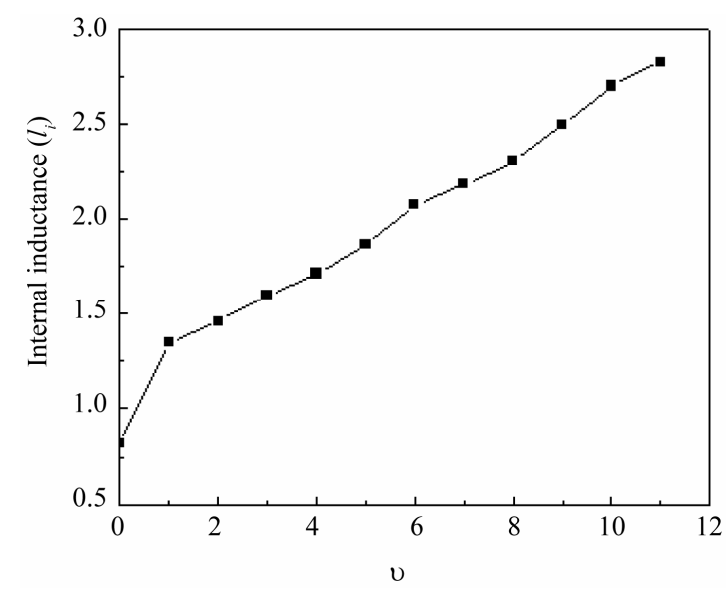

Figure 1. Dependence of the internal inductance $\left(l_{i}\right)$ to the values of $v$ for HT-7 Tokamak plasmas. 
research. Much more plasma parameters such as the plasma current density profile, magnetohydrodynamics instability, and plasma energy confinement time can be determined by using this parameter. Discrete poloidal magnetic probes along with the diamagnetic loop can be utilized in measurement of the Plasma internal inductance $\left(l_{i}\right)$.

Discrete poloidal magnetic probes along with the diamagnetic loop can be utilized in measurement of the Plasma internal inductance $\left(l_{i}\right)$. In this paper Plasma internal inductance $\left(l_{i}\right)$ is studied by theoretical and experimental approach for HT-7 Tokamak plasmas. The results of two methods are in good agreement with each other.

\section{REFERENCES}

[1] M. Asif, et al., Physics Letters A, Vol. 336, 2005, p. 61. doi:10.1016/j.physleta.2005.01.004

[2] M. Asif, et al., Physics of Plasmas, Vol. 12, 2005, Article ID: 082502. doi:10.1063/1.1995627

[3] A. Salar Elahi, et al., Journal Fusion Energy, Vol. 28, 2009, pp. 346-349. doi:10.1007/s10894-009-9198-x

[4] M. Ghoranneviss, et al., Journal Fusion Energy, Vol. 29, 2010, pp. 232-236. doi:10.1007/s10894-009-9264-4

[5] B. Shen, et al., Review of Scientific Instruments, Vol. 12 , 2005, Article ID: 082502.

[6] J. Wesson, "Tokamaks," Clarendon, Oxford, 1997, pp. 105-131. 